\title{
Miranda
}

Revue pluridisciplinaire du monde anglophone /

Multidisciplinary peer-reviewed journal on the English-

speaking world

$18 \mid 2019$

Guerre en poésie, poésie en guerre

\section{Arnaud Dubois, Jean-Baptiste Eczet, Adeline Grand- Clément et Charlotte Ribeyrol (ed.), Arcs-en-ciel et couleurs}

\section{Armelle Sabatier}

\section{OpenEdition}

\section{Journals}

Édition électronique

URL : http://journals.openedition.org/miranda/17573

DOI : $10.4000 /$ miranda. 17573

ISSN : 2108-6559

\section{Éditeur}

Université Toulouse - Jean Jaurès

\section{Référence électronique}

Armelle Sabatier, «Arnaud Dubois, Jean-Baptiste Eczet, Adeline Grand-Clément et Charlotte Ribeyrol (ed.), Arcs-en-ciel et couleurs », Miranda [En ligne], 18|2019, mis en ligne le 16 avril 2019, consulté le 16 février 2021. URL : http://journals.openedition.org/miranda/17573; DOI : https://doi.org/10.4000/ miranda. 17573

\section{Ce document a été généré automatiquement le 16 février 2021.}

\section{cc) (1)}

Miranda is licensed under a Creative Commons Attribution-NonCommercial-NoDerivatives 4.0

International License. 


\section{Arnaud Dubois, Jean-Baptiste Eczet, Adeline Grand-Clément et Charlotte Ribeyrol (ed.), Arcs-en-ciel et couleurs}

Armelle Sabatier

\section{RÉFÉRENCE}

Arnaud Dubois, Jean-Baptiste Eczet, Adeline Grand-Clément et Charlotte Ribeyrol (ed.), Arcs-en-ciel et couleurs (Paris : CNRS Édition, 2018, collection « Bibliothèque de

l'Anthropologie ») $301 \mathrm{p}$, ISBN : 978-2-271-11942-1 


\section{Arcs-en-ciel}

$\&$ couleurs

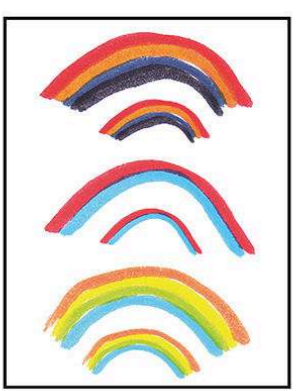

1 « On se doit, à propos de la couleur, de toucher à la philosophie, à la théorie du langage, à la psychologie cognitive, à l'histoire culturelle, à l'histoire des sciences, à l'histoire de l'art. Peu de thèmes réclament autant d'interdisciplinarité ${ }^{1}$ ). Les propos de Jacques Le Rider sur le caractère polymorphe inhérent à toute étude sur la/les couleur(s) sont, encore une fois, parfaitement illustrés par le très bel ouvrage collectif consacré aux liens entre les couleurs et le phénomène météorologique de l'arc-en-ciel. Dans ce volume, des universitaires issus de disciplines différentes ont réuni douze chapitres structurés en triptyque, qui interrogent le lien apparemment indissociable entre l'arcen-ciel et le spectre des couleurs, une construction culturelle profondément ancrée dans nos sociétés occidentales et qui façonne la perception des couleurs chez les Occidentaux depuis presque trois siècles. Une cinquantaine d'illustrations en couleurs, de très grande qualité, viennent étayer les diverses argumentations des auteurs, sans compter de nombreuses reproductions en noir et blanc insérées dans le texte de certains chapitres. Les chapitres, extrêmement savants et rigoureux, comportent, à chaque fois, une bibliographie très riche.

2 Le point de départ de cette étude transversale, qui englobe des sociétés et des siècles très divers, met en lumière le préjugé tenace (même pour les chercheurs avertis qui ont participé à ce volume) selon lequel l'arc-en-ciel est lié à la couleur, plus particulièrement aux sept couleurs mises en évidence par Isaac Newton, dès la fin du XVII ${ }^{\text {ème }}$ siècle, lors de ses expérimentations sur la lumière : cette révolution scientifique a été théorisée dans son Traité d'Optique (1704). Or, comme le souligne l'introduction, l'ordre des couleurs ne correspond pas au phénomène naturel que nous percevons dans le réel: "sa polychromie s'inscrit dans des formes bien définies et obéit à des conventions » (p.7). Le prisme établi par Newton, reposant sur sept couleurs est devenu au fil du temps, et encore de nos jours « l'index des couleurs, voire de la couleur » (p.9). L'équivalence entre le spectre des couleurs et l'arc-en-ciel théorisé par Newton finit par s'imposer au XIX ${ }^{\text {ème }}$ siècle. Néanmoins, les conclusions des travaux scientifiques de 
Newton ont été contestées par de nombreux auteurs, en particulier Johann Goethe qui s'oppose à cette rationalisation des couleurs dans son ouvrage Théorie des couleurs, publié en 1810. Ainsi, à cette époque émergent deux approches différentes de la couleur : celle de Newton qui s'appuie sur les lois de la physique, donc des « couleurs objectives ", et la théorie de Goethe qui défend la perception subjective des couleurs, une perception qui varie d'une culture à une autre ("couleurs subjectives»). Il est à noter que cette division existe encore de nos jours : la perception des couleurs ne se limite pas à la neuroscience, aussi passionnante soit-elle. Notre cerveau est aussi influencé par la culture, les croyances et les coutumes de la société dans laquelle il évolue, comme l'ont déjà démontré de nombreuses études, comme celles de John Gage (Colour and Culture, 1993; Colour and Meaning, 1999) ou encore les volumes consacrés à l'histoire symbolique des couleurs publiés par l'historien médiéviste Michel Pastoureau ${ }^{2}$. L'approche interdisciplinaire des douze études du présent volume confirme cette vision relativiste de la couleur (par opposition à la vision universaliste de la couleur défendue par Berlin et Kay en 1969). La démarche partagée par tous les auteurs consiste à prendre de la distance avec le système newtonien, à "détisser " (pour reprendre la métaphore de John Keats citée dans l'introduction), en quelque sorte, les liens entre arc-en-ciel et couleurs afin d'offrir de nouvelles perspectives sur ce phénomène naturel.

3 Ces regards croisés sur l'arc-en-ciel construisent un nouveau prisme de la couleur en suivant trois directions. La première partie de l'ouvrage remet en question les savoirs et la rationalisation des couleurs de l'arc-en-ciel établis par Newton au travers d'études anthropologiques, linguistiques et littéraires consacrées à des sociétés très éloignées. Ainsi, le chapitre d'Arnaud Dubois ouvre la réflexion avec l'analyse d'une enquête menée par un groupe de chercheurs de Cambridge, en 1898, lors d'une mission d'un an dans le détroit de Torres, situé entre la Papouasie-Nouvelle-Guinée et l'Australie. Le rapport issu de cette mission aborde, entre autres, la question de la perception de l'arcen-ciel chez cette tribu, une perception très différente des Britanniques venus les observer. La société victorienne où les théories du physicien anglais commencent à devenir la norme et qui connaît une révolution dans la production des couleurs avec l'avènement des couleurs de synthèse, rejette, en partie, cette rationalisation du spectre des couleurs. Comme le démontre Charlotte Ribeyrol dans le chapitre 4, les poètes anglais tels que John Keats ou encore Oscar Wilde, refusent de réduire la couleur à une rationalisation scientifique qui ne rend pas compte de la complexité et de la diversité de la perception humaine des couleurs. Les deux autres chapitres de cette première partie commencent à annoncer les constats de la deuxième partie. L'étude sur le Japon ainsi que celle consacrée aux Mursi, une tribu composée d'agropasteurs transhumants du Sud-Ouest éthiopien, détissent l'arc-en-ciel newtonien. Dans son chapitre sur le Japon (du XVII ${ }^{\mathrm{ème}}$ au XIX ${ }^{\text {ème }}$ siècles), Marie Parmentier démontre que les théories scientifiques, entre autres celles de Newton, imposées sous l'ère Meiji à la société japonaise, n'ont pas pour autant supplanté des croyances plus anciennes qui subsistent encore aujourd'hui.

4 C'est surtout dans la deuxième partie que le postulat newtonien vacille véritablement dans la mesure où, dans les sociétés antiques, ce phénomène météorologique pouvait être tout simplement invisible. Ce paradoxe est savamment expliqué par Sylvie Donnat dans son chapitre centré sur l'Égypte ancienne : l'arc-en-ciel serait inexistant à cette époque, du moins dans les textes disponibles à ce jour. L'auteur avance une raison purement météorologique, à savoir que ce phénomène naturel est plutôt rare dans ces 
régions. Ainsi, il est très difficile pour le chercheur de comprendre si et comment les Égyptiens pouvaient percevoir l'arc-en-ciel. Néanmoins, d'autres phénomènes fascinaient davantage les Égyptiens comme l'aube, associée à la couleur rouge (p.164). C'est d'ailleurs dans ce ton que le poète Homère décrivait l'arc-en-ciel, semblable à la très précieuse couleur pourpre. Adeline Grand-clément souligne d'ailleurs une opposition, dans la Grèce antique, entre la description poétique de l'arc-en-ciel et le discours scientifique défendu par Aristote pour qui l'arc-en-ciel était trichrome et se déclinait en rouge, vert et un violet se rapprochant du pourpre. Un autre chapitre de cette partie est consacré au monde préhispanique. Le Codex de Florence qui rassemble les observations d'un frère franciscain auprès de la tribu des Nahuas établie dans les hautes terres du Mexique Central dévoile que, dans cette culture, l'arc-en-ciel est souvent associé au vent.

La troisième et dernière partie de l'ouvrage invite le regard du lecteur à observer le paradoxe constitutif de toute représentation visuelle de l'arc-en-ciel qui consiste à fixer un phénomène insaisissable et éphémère. Cette réflexion s'ouvre, bien entendu, sur les représentations bibliques de ce phénomène naturel. François Jacqueson rappelle, à juste titre, que dans l'Ancien Testament, l'arc-en-ciel symbolise l'alliance entre Dieu et les hommes (Genèse $9: 12-15)$. Dans le livre sacré, ce n'est pas la couleur qui est mise en avant, mais sa forme, à savoir l'arc. Au travers de mosaïques et de fresques du Moyen Âge, magnifiquement reproduites dans l'ouvrage, l'auteur dévoile les diverses formes de ce phénomène naturel dans les représentations religieuses. Aux mosaïques succèdent les tableaux du peintre anglais John Constable analysés par Jean-Loup Korzilius : un retour vers le XIX ${ }^{\text {ème }}$ siècle anglais et ses interrogations sur la lumière et les couleurs. Les deux derniers chapitres explorent les représentations de l'arc-en-ciel dans les arts visuels contemporains, en particulier dans un film d'animation de Len Lye, Rainbow Dance (1936).

\section{NOTES}

1. Jacques Le Rider, Les Couleurs et les mots, Paris : Presses Universitaires de France, 1997, p.4.

2. Cet historien a publié aux éditions du Seuil des ouvrages sur l'histoire symbolique des couleurs suivantes : Bleu, Histoire d'une couleur (2002), Noir, Histoire d'une couleur (2008), Vert, Histoire d'une couleur (2013), Rouge, Histoire d'une couleur (2016). Un volume sur le jaune devrait être publié prochainement. 
INDEX

Keywords : anthropology, Bible, colours, Egypt, Ethiopia, Japan, light, purple, Quetzalcoatl, rainbow, Torres strait

Mots-clés : anthropologie, arc-en-ciel, Bible, couleurs, détroit de Torres, Égypte, Éthiopie, Japon, lumière, pourpre, Quetzalcoatl

\section{AUTEURS}

\section{ARMELLE SABATIER}

Maître de conférences

Université Panthéon - Assas (Paris 2)

arm.saba@free.fr 